OBETS. Revista de Ciencias Sociales

Vol. 16, no 2, 2021, pp. 263-280

ISSN: 1989-1385

https://doi.org/10.14198/OBETS2021.16.2.03

\title{
CONSUMO ALIMENTARIO Y SALUD: HÁBITOS ALIMENTARIOS DEL COMENSAL EXTRADOMÉSTICO ESPAÑOL
}

\author{
FOOD CONSUMPTION AND HEALTH: EATING HABITS OF A SPANISH \\ EXTRA-DOMESTIC DINER
}

\author{
Vidal Díaz de Rada \\ Universidad Pública de Navarra, España \\ vidal@unavarra.es \\ https://orcid.org/0000-0002-9638-3741 \\ Cecilia Díaz-Méndez \\ Universidad de Oviedo, España \\ cecilia@uniovi.es \\ https://orcid.org/0000-0003-3200-4922
}

Cómo citar / Citation: Díaz de Rada, V. y Díaz-Méndez, C. (2021) "Consumo alimentario y salud: Hábitos alimentarios del comensal extradoméstico español”. OBETS. Revista de Ciencias Sociales, 16(2): 263-280. https://doi.org/10.14198/OBETS2021.16.2.03

\section{(C) 2021 Vidal Díaz de Rada, Cecilia Díaz-Méndez}

Este es un artículo de acceso abierto distribuido bajo los términos de la licencia de uso y distribución Creative Commons Reconocimiento 4.0 Internacional (CC BY 4.0) https://creativecommons.org/licenses/by/4.0/deed.es

Recibido: 15/01/20. Aceptado: 10/03/21

\section{Resumen}

La creciente importancia de la alimentación fuera del hogar es un hecho constatado y un rasgo característico de la modernidad. La literatura especializada asocia el consumo extradoméstico de alimentos a un perfil específico de comensal vinculando esta práctica a los ingresos, al nivel educativo y a la ocupación. Este trabajo sigue en esta línea para corroborar el perfil del comensal extradoméstico español, aportando como novedad, por un lado, un aumento en el número de variables y, por otro, un cambio en la temática de estas variables. Esto es posible gracias a la incorporación de preguntas relacionadas con la alimentación fuera del hogar en los Barómetros del Centro de Investigaciones Sociológicas (CIS, 2017). A las "tradicionales" variables sociodemográficas se añaden siete variables relacionadas con diferentes hábitos de la vida cotidiana y con incidencia sobre la salud de los individuos: el horario de levantarse y el de acostarse, el número de comidas en días laborables, el consumo de diferentes alimentos, la frecuencia de comidas fuera de casa, la práctica de ejercicio físico, y la autovaloración de su estado de salud. Los resultados de esta investigación muestran que el perfil del comensal fuera de casa presenta una dieta diferente al comensal doméstico, duerme menos horas y hace menos

\begin{abstract}
Eating out is a food behavior that has increased in modern societies and has been modified in the modernization process. The specialized literature associates the extra-domestic food consumption with a specific social profile, linking this practice with income, educational level, and occupation. This work continues along this line to corroborate the profile of the Spanish extra-domestic diner, contributing as a novelty, on the one hand, an increase in the number of variables and, on the other, a change in the theme of these variables. This is possible thanks to the incorporation of questions related to eating outside the home in the Barometers of the Sociological Research Center (CIS, 2017). To the "traditional" sociodemographic variables are added seven variables related to the different habits of daily life and their impact on people's health: time to get up and go to bed, number of meals on weekdays, consumption of different foods, the frequency of meals away from home, physical activity, and self-assessment of your health. The results of this research show that the profile of the diner outside the home has a different diet than the domestic diner, sleeps fewer hours, and does less sport than the average of the Spanish population. Despite these
\end{abstract}


deporte que la media de la población española. A pesar de estas diferencias, el nivel educativo sigue siendo la variable principal a la hora de explicar este hábito alimentario.

Palabras clave: Consumo alimentario fuera del hogar; consumo extradoméstico y salud; análisis de segmentación; sociología de la alimentación.

\section{Extended abstract}

The results show a profile of extra-domestic diner that differs from the average Spanish in several aspects: we are dealing with a male, under 44 years of age and among those who are predominantly employed people with a high educational level. Although a differentiated profile is detected that distances it from the rest of the population, we are not facing a diner with drastically different habits from the rest, but they do differ in some eating habits that can be significant since they consume more meat, fewer vegetables and legumes, fewer fruits, more eggs and sweeter than the rest of the population. All this makes your daily diet present less healthy habits. This is accompanied by a daily sports practice less than that of the majority of the population. In addition, they wake up later and go to bed later than most, so the hours of sleep are less. Despite these traits, which could bring us closer to less healthy habits, extra-domestic diners have a more positive self-assessment of their health than that of the rest of the population.

Various studies have indicated that food expenditure outside the home has a direct relationship with income, occupation, and educational level. Now, what differentiates this work from the previous research is that here all these variables, along with others related to daily healthy habits, act simultaneously to explain the habit of eating outside the home. The results indicate that the educational level is the variable that has the greatest explanatory power. The second variable with the greatest explanatory power is age, in people with primary education, and in those with second stage and higher secondary education. In the group with first-stage secondary studies, the relationship with activity is the second most influential variable.

In the third level of influence, there are several variables with less explanatory power: sex in people with primary education aged 65 and over; the age at which they have first-stage secondary studies and work/study, the frequency of physical exercise in the rest of the people with first-stage secondary studies (retired, housewives and unemployed) differences, the educational level continues to be the main variable to explain this eating habit.

Keywords: Eating out; eating out and health; segmentation analysis; food sociology.

and the relationship with the activity in interviewees with more than first-stage primary studies and between 25 and 34 years. In short, the results reveal, in particular, the great segmentation power of the educational level, thus coinciding with other previous works and reinforcing the importance of sociodemographic variables.

Finally, two aspects of the results should be qualified: on the one hand, the weight of education could be indicating that greater knowledge, more information or greater receptivity to messages modifies food choices and determines eating outside the home, however, It can also be assumed that this variable is associated with income, thus, it would not be so much the training as the income that facilitates qualification, which would explain the differences in behavior. On the other hand, the confirmation that the sociodemographic variables are more explanatory than the variables related to healthy lifestyles may be indicating that they mark the extradomestic diet, but also that the health variables associated with daily life do not reflect differences between extradomestic and domestic eating behaviors. As seen in other studies, eating habits in Spain, in both areas, do not differ either in the type of food eaten, or in the schedules, or in the company (Díaz-Méndez and García Espejo, 2017). We are facing behaviors deeply rooted in Spanish food culture.

The main contributions of this work, noting the great explanatory power of the sociodemographic variables, compared to those related to daily life such as hours of sleep, the number of meals eaten on weekdays, the consumption of different foods, the frequency of meals away from home, the practice of physical exercise, and self-assessment of their health. Although various studies have highlighted that these are variables that influence in the health of individuals, the joint analysis of these variables and the sociodemographic variables reveals a lesser influence of the former. In short, the present work corroborates the profile of the Spanish extradomestic diner, with little influence from the variables of daily life.

\section{EL CONSUMO ALIMENTARIO FUERA DEL HOGAR EN ESPAÑA}

Los estudios que analizan la alimentación fuera del hogar en España confirman, ya en los últimos años del siglo XX, que nos encontramos ante una tendencia contraria al consumo doméstico, al menos en términos de gasto. Mientras el presupuesto que los hogares dedican a la alimentación dentro del hogar desciende, el extradoméstico crece. El sector de la restauración muestra los signos de este crecimiento y se ha convertido en un importante motor de la economía española, tanto en términos de empleo como de facturación (Martín 
Cerdeño, 2017). En cuanto al primero, más de 140.000 personas están empleadas en la restauración, sector que tiene una facturación superior a los 10.000 millones de euros anuales (Asociación Nacional de Marcas de Restauración, 2018), con un elevado crecimiento continuo desde el año 2001 (Observatorio Sectorial DBK, 2017). Entre los responsables de esta situación está el mayor número de turistas, evidentemente, pero muy especialmente el aumento del gasto extradoméstico de los hogares (Martín Cerdeño, 2017 y 2012; Encuesta de Presupuestos Familiares, 2017).

Los datos animan a analizar las variables sociodemográficas para buscar explicaciones sobre este crecimiento. Se confirma que el aumento del gasto doméstico en la alimentación fuera del hogar está directamente ligado a la renta, de tal modo que el aumento de los ingresos familiares va acompañado de un aumento del gasto alimentario extradoméstico. Esta relación se ha confirmado en España (Rama, 1997) y en otros países (Warde y Martens, 2000). En estos contextos de países desarrollados, las clases sociales más altas son las que dedican una mayor partida presupuestaria a la alimentación fuera del hogar, así como las responsables principales del crecimiento de la restauración comercial.

La ocupación explica una buena parte de este aumento de la renta, y por tanto del aumento del consumo. En el caso español se asoció este aumento de la renta al trabajo externo remunerado de las mujeres (Rama, 1997). En el Reino Unido Cullen (1994) constata que en los últimos años de la década de los ochenta el gasto en la alimentación fuera del hogar se trasladó de manera progresiva del restaurante tradicional al establecimiento de comida rápida. En ambos casos, tanto en España como en Reino Unido, el aumento del gasto extradoméstico es interpretado como un signo de la relación entre el consumo y el empleo. Se entiende que el aumento del gasto en alimentación fuera del hogar es resultado del aumento de la renta del hogar que hace valorar los menores costes y mayores beneficios de la comida fuera de casa, en especial por parte de las mujeres trabajadoras.

Los estudios de Gofton (1995) sobre el consumo de alimentos de conveniencia y el eating out en una población formada por mujeres y adolescentes, confirman que la variable género y la edad también guardan relación con el consumo alimentario. Las mujeres que consumen más alimentos fuera del hogar son las que están expuestas a las nuevas exigencias temporales que marca el mercado laboral. En España, en esa misma década, también se confirma que el consumo alimentario fuera del hogar es mayor en los hogares de rentas altas, aunque también está ligado a la ocupación, la edad, el nivel de ingresos o los estudios: Gastan más en alimentación fuera de casa los ocupados, los que viven solos, los jóvenes o personas de mediana edad sin niños, y las parejas con un hijo (Rama, 1997).

La importancia de la ocupación va cobrando peso en España a medida que se analizan datos más recientes. Martín Cerdeño (2003) confirma que, si bien el consumo de alimentos en el hogar disminuye a medida que se aumenta en la jerarquía ocupacional, en el caso de la alimentación extradoméstica la tendencia es la contraria: Son las personas que ocupan categorías ocupacionales altas las que más gastan en alimentación fuera del hogar.

Algunos autores han avanzado en el análisis de las variables sociodemográficas y han comparado el consumo extradoméstico fuera del hogar en contextos sociales distintos, confirmando algunas diferencias significativas que animan a explorar este comportamiento alimentario en relación directa con sus entornos de consumo. Es el caso de la comparación que Díaz Méndez y García Espejo (2012) realizan sobre el gasto extradoméstico en Reino Unido y España.

Hay similitudes, pues en ambos países es importante el nivel educativo, la composición del hogar y la edad para explicar este gasto: los de niveles educativos más altos, los hogares en los que hay al menos dos adultos y los de edades intermedias o más jóvenes. Pero también importantes diferencias en relación con la ocupación, pues mientras en el caso español es la ocupación la que establece diferencias a favor de los ocupados, sea cual sea su categoría ocupacional, son los parados británicos los que presentan una asociación positiva con la comida fuera del hogar, superior incluso a los de categorías ocupacionales más altas (Díaz Méndez y García Espejo, 2013).

Una práctica menos instrumental y más orientada al ocio y a las relaciones ha sido otra de las explicaciones esgrimidas del aumento de la comida fuera de casa. Siguiendo a Bourdieu (1991) su práctica sería un rasgo de distinción de ciertos grupos sociales que expresan su capital cultural y sus gustos a través de la elección de 
restaurantes (McCracken y Brandt, 1987; Riley, 1994; Warde y Martens, 2000; Olsen, Warde y Martens, 2000; Bojanic y Xu, 2006; Cheng et al., 2007; Díaz-Méndez y García-Espejo, 2013). En esta línea interpretativa se confirma que los comensales valoran muy positivamente la comida fuera del hogar considerándola una experiencia gratificante (Warde y Martens, 1998, 2000). El nivel educativo es, así, otra de las variables explicativas de la alimentación fuera del hogar.

Como se ha visto, las variables sociodemográficas permiten explicar el consumoalimentario fuera del hogar. Este trabajo sigue en esta línea para corroborar el perfil del comensal extradoméstico, aportando como novedad, por un lado, un aumento en el número de variables y, por otro, un cambio en la temática de estas variables. A las "tradicionales" variables sociodemográficas, muy presentes en la literatura, se añaden siete variables relacionadas con hábitos de la vida cotidiana y cuya incidencia sobre la salud de los individuos ya ha sido corroborada: El horario de levantarse y acostarse, el número de comidas en días laborables, el consumo de diferentes alimentos, la frecuencia de comidas fuera de casa, la práctica de ejercicio físico, y la autovaloración de su estado de salud.

El número de horas de sueño (obtenido a partir de la hora de levantarse y acostarse) se ha confirmado como una variable directamente relacionada con la salud (Åkerstedt et al., 2019). Se ha visto, además, su relación con la alimentación confirmándose que una reducción en las horas de sueño induce a una mayor ingesta de alimentos y que los patrones de sueño cortos están relacionados con el sobrepeso y la obesidad (Escobar et al., 2013; Marqueta se Salar et al., 2017).

Respecto al número de comidas, la frecuencia de consumo de alimentos y la práctica del ejercicio físico, son variables comunes en los estudios sobre los hábitos alimentarios de la población (Serra Majem et al., 2000; Estudio ALADINO, 2015). Con datos no siempre concluyentes en relación con la obesidad, sí se ha confirmado su relevancia para conocer los hábitos alimentarios y se aborda el estudio de la alimentación fuera del hogar bajo la hipótesis de una ingesta calórica mayor. Estas variables están integradas en las encuestas nacionales de salud españolas como variables determinantes de los hábitos alimentarios (ENS).

En relación con la autovaloración sobre la propia salud y sus efectos sobre ésta, los estudios son muy abundantes desde hace décadas (Castro-Vazquez et al., 2007; Diener et al., 1999; Girón, 2010) pero no lo son tanto los que se ocupan de la relación entre la percepción subjetiva de salud y la alimentación (Veenhoven, 2019). En el caso español se ha visto que, en algunos grupos sociales se produce un seguimiento más correcto de las dietas saludables (como la mediterránea) cuando hay una mejor valoración de la salud percibida, pero esto no se ha corroborado en todos los casos (Abellan et al., 2016; Ferrer-Cascales et al., 2019; Grao-Cruces et al, 2002). Lo que sí se puede confirmar, a través de investigación cualitativa, es la importancia que se le da a la comida fuera de casa como referente de sociabilidad, hasta el punto de considerar que es un hábito que se resiste a perecer incluso en tiempos de crisis y entre población con menores recursos (Ramos Truchero y Castaño, 2018; Truninger y Vasco, 2021).

La hipótesis principal de este trabajo es que estas siete variables, todas ellas asociadas con la salud y la alimentación, permiten complementar las explicaciones sobre el comensal extradoméstico al ampliar la información procedente las variables sociodemográficas tradicionales. Esto ofrece un perfil más preciso sobre este comensal, en tanto en cuanto no solo se ofrece información sobre un comportamiento alimentario, sino también sobre la importancia que tienen los aspectos relacionados con la salud en este hábito de consumo.

\section{OBJETIVOS Y METODOLOGÍA}

El trabajo estudia hasta qué punto un conjunto de (siete) variables relacionadas con hábitos de la vida cotidiana, y cuya incidencia sobre la salud de los individuos ya ha sido corroborada, permiten complementar la explicación de las tradicionales variables sociodemográficas. Estas variables son el horario de levantarse, de acostarse, número de comidas en días laborables, consumo de diferentes alimentos, frecuencia de comidas fuera de casa, práctica de ejercicio físico, y autovaloración de su estado de salud. La incidencia de algunas de estas sobre la salud de los individuos ya ha sido corroborada, pero en la mayor parte de las ocasiones se trata de estudios que no consideran conjuntamente las variables aquí utilizadas. La influencia de estas variables permitirá responder a un segundo objetivo, elaborar el perfil del comensal extradoméstico, 
Con el fin de resolver estos objetivos, y resolver así la hipótesis planteada, se utilizará el Barómetro de Centro de Investigaciones Sociológicas (en adelante CIS), concretamente el Barómetro de marzo de 2017 (estudio CIS $n^{\circ}$ 3170). Esta fuente cuenta con una pregunta básica sobre la alimentación fuera del hogar en la que se solicita información acerca de la frecuencia de consumo ofreciendo tres opciones: comer en un restaurante, cafetería o similar; comer en casa de familiares o amigos; y comer fuera de casa alimentos preparados (en casa o comprados). Además de esta pregunta sobre la alimentación, el Barómetro solicita información sobre la frecuencia de consumo de un grupo de alimentos, utilizando los mismos productos y la misma escala que la utilizada en la Encuesta Nacional de Salud (en adelante, ENS), y sobre el número de comidas que se realizan al día.

Contempla también otros aspectos que, si bien no están directamente relacionados con la alimentación, son fundamentales para el conocimiento de la vida cotidiana de la población española: frecuencia con la que se hace ejercicio y el número de horas de sueño, incluidos también en la ENS (CIS, 2017. Metodología). En el cuadro 1 se pueden ver las preguntas del cuestionario que se han seleccionado para este trabajo.

Cuadro 1. Sobre aspectos relacionados con los hábitos de la vida cotidiana incluidos en el estudio.

\begin{tabular}{|c|c|}
\hline Preg. 12. & $\begin{array}{l}\text { Horario habitual para acostarse } \\
\text { en días laborables. }\end{array}$ \\
\hline Preg. 13. & $\begin{array}{l}\text { Horario habitual para levantarse } \\
\text { en días laborables. }\end{array}$ \\
\hline Preg. 14. & $\begin{array}{l}\text { Número habitual de comidas } \\
\text { en un día laborable. }\end{array}$ \\
\hline Preg. 15. & Frecuencia de consumo de diferentes alimentos. \\
\hline Preg. 17. & $\begin{array}{l}\text { Frecuencia con la que se hacen } \\
\text { comidas fuera de casa }\end{array}$ \\
\hline Preg. 18. & Frecuencia en la práctica de ejercicio físico \\
\hline \multirow[t]{2}{*}{ Preg. 22.} & Valoración de su estado de salud en general \\
\hline & Aspectos sociodemográficos \\
\hline \multicolumn{2}{|c|}{$\begin{array}{l}\text { - Sexo } \\
\text { - Edad en cuotas: } 18 \text { - } 24 \text { años, } 25 \text { - 34, } 35 \text { - 44, } 45 \text { - 54, } 55 \text { - } 64 \\
\text { y } 65 \text { y más años } \\
\text { - Nivel de estudios del entrevistado: Primarios, Secundarios de: } 1^{\text {a }} \\
\text { etapa, secundarios de } 2^{a} \text { etapa, Formación Profesional, estudios } \\
\text { universitarios medios y superiores. } \\
\text { - Relación con la actividad: Ocupado, Parado, Jubilado, Estudiante } \\
\text { y Trabajos domésticos no remunerados } \\
\text { - Status del hogar: Clase media-alta, Nuevas clases medias, } \\
\text { Viejas clases medias, Obreros/as cualificados/as, Obreros/as no } \\
\text { cualificados/as }\end{array}$} \\
\hline
\end{tabular}

Fuente: Centro de Investigaciones Sociológicas, Barómetro de marzo 2017, estudio 3170
Las variables sociodemográficas son las habituales en este tipo de estudios (sexo, edad, nivel de estudios y relación con la actividad), a las que se añade una variable adicional: el estatus socioeconómico del hogar. Se trata, como explica dice García-Pardo (1998) de un modelo de clase weberiano que considera la actividad profesional, frente a la propiedad. Para su formulación se considera la ocupación con características homogéneas en la educación e ingresos de los entrevistados. En la definición de la clasificación se utiliza la situación laboral (ocupado, parado e inactivo), dependencia laboral (cuenta propia o ajena), ocupación (Clasificación Nacional de OcupacionesCNO a tres dígitos), rama de actividad, tamaño de la empresa, educación y nivel de estudios.

A los no ocupados se les asigna el status de la persona del hogar que recibe mayores ingresos (García Pardo, 1998: 60 $)$. En el cuadro 2 se muestran las ocupaciones incluías en cada grupo. Terminar esta explicación señalando que es una variable incluida en todos los barómetros del CIS.

Cuadro 2. Ocupaciones incluidas en cada agrupación.

- Clase alta/media alta Profesionales y técnicos, directivos y cuadros medios, técnicos y profesionales subordinados.

- Nuevas clases medias Asalariados no manuales, administrados de nivel inferior, trabajadores de cuello blanco.

- Viejas clases medias Empresarios, autónomos, agricultores.

- Obreros cualificados Obreros manuales, cualificados, semicualificados, capataces y artesanos.

- Obreros no cualificados Profesionales y técnicos, directivos y cuadros medios, técnicos y profesionales subordinados.

Fuente: reproducido de García Pardo, 1998: 60.

El universo objeto de estudio es la población española de 18 y más años (excepto los residentes en Ceuta y Melilla) que es entrevistada en su domicilio en presencia del encuestador. En los diez primeros días de marzo del 2017 fueron entrevistadas 2.487 personas utilizando un diseño muestral en varias etapas, en el que las unidades primarias (municipios) y las unidades

1 Una explicación más pormenorizada de la construcción del índice se muestra en CIS (año desconocido), y en ttp://www. cis.es/cis/opencms/ES/3_publicaciones/Anuarios/Listado/2016/ Metodologia.html. 
secundarias (secciones) fueron seleccionadas de forma aleatoria proporcional, y las unidades últimas (individuos) por rutas aleatorias y cuotas de sexo y edad. Para un nivel de confianza del 95,5\% (dos sigmas), y $\mathrm{P}=\mathrm{Q}$, los datos tienen un error muestral del $\pm 2,0 \%$ para el conjunto de la muestra y en el supuesto de muestreo aleatorio simple (CIS, 2017. Metodología).

El análisis será, en una primera fase, de carácter descriptivo, con objeto de realizar una aproximación general a los resultados y presentar el perfil del comensal extradoméstico español. En la definición de tal perfil se consideran variables sociodemográficas (gráfico 2) y otras de comportamiento en relación a las horas de sueño (hora habitual de levantarse y acostarse), práctica de deporte, autovaloración de la salud, y consumo de determinados alimentos (gráficos 2 y 3 ). En un segundo momento, se utilizará el estadístico $V$ de Cramer, que señala la magnitud de la asociación entre dos variables con el objetivo de conocer la influencia de estas variables en la frecuencia de comer fuera de casa. En un tercer momento se empleará un Análisis Automático de la Interacción (Sonquist y Morgan, 1964) o Análisis de Segmentación, con el fin de considerar qué variables son más determinantes en el consumo alimentario fuera del hogar; las sociodemográficas o las variables que describen comportamientos relacionados con la vida cotidiana. Esta técnica permitirá elaborar tipologías de entrevistados/as. Se trata, en definitiva, de averiguar si es posible identificar segmentos de población dentro de los cuales la variación de la comida fuera del hogar sea significativamente menor, o mayor, que en el conjunto de la muestra. Teniendo en cuenta que la mayor parte de las variables de esta investigación presentan un nivel de medición nominal, se utilizará una versión del AID denominada CHAID-Chi-Square Automatic Interaction Detector (Kass, 1980; Magdison \& Vermut, 2005). Román y Levy (2003) señalan algunas ventajas que justifican la adecuada en el empleo de esta técnica.

- Identifica las categorías de variable semejantes respecto a la variable a explicar, uniéndolas en una misma categoría. Así supera la tradicional partición dicotómica del AID, manteniendo separadas las categorías que son heterogéneas respecto a la variable a explicar.

- Permite la eliminación segura de variables no significativas.
- Considera la relación existente entre las variables predictoras. Cuanto existe relación entre un grupo de variables selecciona únicamente una de ellas, en la medida que esta ha recogido el efecto del resto, lo que supone "una unión de variables desde el punto de vista explicativo" (Román y Levy, 2003: 372).

Frente al análisis "variable a variable" de la V de Cramer, esta técnica presenta un avance importante en la medida en que considera cómo un conjunto de variables ("predictoras") generan grupos homogéneos sobre una variable dependiente "de contenido". De las diversas interpretaciones que se pueden realizar de la estructura arbórea resultante del análisis de segmentación (Escobar 1998 y Escobar 2007), este trabajo considerará el orden en el que las variables son introducidas en el análisis, puesto que el interés se centra en conocer en qué medida contribuye cada una de las variables independientes en el comer fuera del hogar. En un segundo momento, la interpretación se centrará en las asociaciones entre variables y categorías de variables, esto es, en el análisis de los "tipos" resultantes mediante las combinaciones de variables.

\section{FRECUENCIA DE COMIDAS FUERA DEL HOGAR}

La fuente de datos utilizada desvela que la conducta de comer fuera del hogar es muy heterogénea y cuenta con una variada diversidad de opciones. Esta fuente distingue entre comidas en restaurantes y cafeterías, comidas en casas de familiares o amigos, y comida traída de casa o comprada ya manufacturada para su consumo fuera del hogar. La pregunta ofrece siete posibles respuestas ('Casi todos los días', 'Varias veces a la semana', 'Varias veces al mes', Una vez al mes', 'Varias veces al año?, 'Una vez al año' y 'Casi nunca o nunca'. Basados en motivos prácticos, debido al escaso número de personas que eligen las opciones extremas (tanto de mayor como menor consumo), se ha decidido realizar una unión de categorías generando así cuatro situaciones de consumo: diario, semanal, mensual y excepcional (Gráfico 1). Concentraremos nuestro análisis en aquel hábito ligado a las comidas en restauración comercial, es decir, en la clasificación de restaurantes, cafeterías, etc.

2 Las referencias a las categorías de respuestas se realizarán, a partir de este momento, con una comilla, utilizando dos comillas (") cuando se trate del texto de preguntas. 
El comensal extradoméstico se distribuye del siguiente modo en función de su frecuencia de consumo fuera del hogar y se pueden observar los porcentajes en el Grafico 1:

a) Frecuencia alta, o consumo Diario: el $12 \%$ de los españoles come con esta alta regularidad en restaurantes/cafeterías pues un 9,1\% lo hace 'varias veces a la semana' y un 2,4\% lo hace 'casi todos los días'.

b) Frecuencia media/semanal: Un $27 \%$ de los entrevistados come en restaurantes/cafeterías 'varias veces al mes', porcentaje que ha aumentado en cinco puntos desde 2014 (CIS, 2014).

c) Frecuencia baja/mensual: La mayoría de los españoles tiene una frecuencia baja de consumo extradoméstico. Concretamente un $41 \%$ de la sociedad española acude a comer a restaurantes/ cafeterías con una frecuencia baja, bien 'una vez al mes' o 'varias veces al año' (20,4\% y 20,6\% respectivamente). Estas cifras han aumentado cuatro puntos porcentuales en los últimos tres años; debido al incremento en la categoría "una vez al mes' (CIS, 2014).

d) Excepcional: Uno de cada cinco españoles apenas acude a comer a restaurantes/cafeterías teniendo, por tanto, una frecuencia excepcional de este tipo de comidas (20,6\% lo hace 'una vez al año' o 'nunca'). Esta cifra se ha reducido nueve puntos cuando se compara con los registros del año 2014 (CIS, 2014).

Gráfico 1: Frecuencia con la que se come fuera de casa en diferentes espacios.

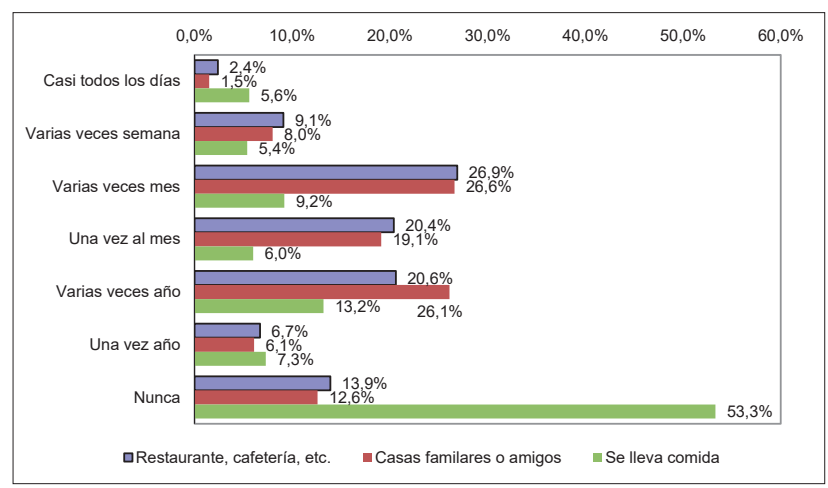

Fuente: Elaboración propia con datos del Centro de Investigaciones Sociológicas, estudio $n^{\circ} 3170$, pregunta 17: frecuencia con la que come y/o fuera de casa, en casa de familiares o amigos/as, o se lleva o compra comida para comerla fuera de casa.
La comida en casa de familiares y amigos es menos habitual. Aunque la realiza un 9,5\% (1,5\% + 8\%) de españoles con una frecuencia alta, el doble (18,7\%) lo hace de modo excepcional. La tercera de las situaciones consideradas, "llevarse comida de casa o comprar comida para llevar fuera de casa", denominada conjuntamente "comida en el puesto de trabajo", presenta una menor frecuencia, puesto que algo más de la mitad de la muestra, un 53,3\% 'nunca' lo hace. Esta cifra aumenta al 60\% cuando se agregan las dos últimas categorías ('nunca' y 'una vez al año'). Se trata de un comportamiento que ha descendido 10 puntos en los últimos cinco años, algo sorprendente cuando se releen investigaciones que -hace menos de un quinquenioconsideraban que "llevar la fiambrera a la oficina" será un hábito al alza en la sociedad post-crisis, hábito que "reemplazará" la comida en restaurantes de menú a mediodía (Nueno, 2015). Sin confirmar esta situación, sí se ha detectado en otros estudios que esta práctica no está generalizada y es propia de algunos colectivos concretos, los jóvenes estudiantes universitarios (DíazMéndez, 2013).

Los datos del grafico 1 muestran que un $11 \%$ de los entrevistados comen diariamente en el puesto de trabajo, algunos menos llevan a cabo un consumo semanal (9,2\%), aumentando el consumo mensual casi hasta el $20 \%$.

Por un lado, es importante recordar que existen algunos problemas en los conceptos que se cuantifican como alimentación fuera del hogar que dificultan la interpretación de los resultados. En primer lugar, condiciona la interpretación la distribución del lugar de consumo englobado en el apartado de "restaurantes, cafetería, etc." Siguiendo los datos del consumo extradoméstico ofrecidos por el Ministerio de Agricultura, Alimentación y Medio Ambiente se puede afirmar que el consumo extradoméstico más habitual se hace, precisamente, en restaurantes y cafeterías, alcanzando al 63\% del consumo fuera del hogar (Ministerio de Agricultura, Alimentación y Medio Ambiente, 2016). Según esta fuente, el 33,2\% eligen bar/cafetería y cervecería, y el 31,2\% restaurantes. Hay, no obstante, un $17,1 \%$ de españoles que optan por restaurantes de comida rápida, y otro $7,9 \%$ que optan por canales diversos como panaderías, pastelerías, máquinas de vending, tiendas de alimentación, etc. El $10 \%$ señalan otros canales. 
Por otro lado, cabe insistir en la gran "amplitud" del segundo de los comportamientos, la comida con familiares y amigos, que identifica los comensales pero no los anfitriones, información imprescindible para poder conocer si se trata de un "comensalismo parasitario" (donde los invitados y anfitriones son siempre los mismos) o más bien de un "comensalismo en círculo", donde ambos -invitados y anfitrionescambian sus roles con frecuencia (Callejo, 2017: 137).

\section{DIFERENCIAS EN EL COMPORTAMIENTO DE LOS COMENSALES EXTRADOMÉSTICOS Y LA POBLACIÓN GENERAL}

Con el fin de concretar las características de las personas que comen fuera del hogar en España, y así aproximarnos a la comprensión de este comportamiento de consumo, el estudio se centrará en el consumo extradoméstico más habitual y que cuenta con una mejor aproximación conceptual en el Barómetro del CIS: el consumo extradoméstico realizado en establecimientos de restauración comercial (denominado en el Barómetro "restaurantes, cafeterías, etc."), y -más concretamenteen los sujetos que lo realizan con una frecuencia alta y media (todos los días, varias veces a la semana, y varias veces al mes). Esto supone, considerando los datos del gráfico 1, algo más de un tercio de la población española (un 38,4\%). Algunos autores han denominado a este comensal como relacional (Callejo, 2017:139), al acudir al restaurante con el fin de encontrarse con amigos o familiares.

Esto no implica la eliminación de las otras dos categorías, la que engloba al consumidor que come fuera de casa 'una vez al mes' y consumidor excepcional ('varias veces al año' 'una vez al año', 'nunca'), pues los análisis se realización considerando el contraste con aquellos que frecuentan menos estos establecimientos. Ahora bien, los propios objetivos de este trabajo llevarán a la descripción de los primeros.

La caracterización sociodemográfica (Gráfico 2) da cuenta de un perfil de comensal extradoméstico que se diferencia de la media de la sociedad española, pues se localiza una mayor presencia de hombres (56\%), el $58 \%$ son menores de 44 años $(13+22+23)$ y casi dos de cada tres $(62 \%)$ están ocupados. Cuando se considera el nivel educativo y la clase social se aprecia mayor presencia de personas con estudios universitarios entre los comensales extradomésticos (35\%) y pertenecientes a las clases altas y medias altas (31\%). Son resultados que se muestran en línea con otros trabajos realizados en España que dan cuenta que el gasto extradoméstico está directamente ligado a la ocupación y al nivel educativo, tratándose de una práctica que aumenta notablemente en las personas que trabajan y en los universitarios (Díaz Méndez y García Espejo, 2017; Fundación Mapfre, 2015).

Gráfico 2: Rasgos sociodemográficos de las personas que comen en restaurantes/cafeterías con una frecuencia diaria (alta frecuencia) y semanal (frecuencia media), y distribución de la población española

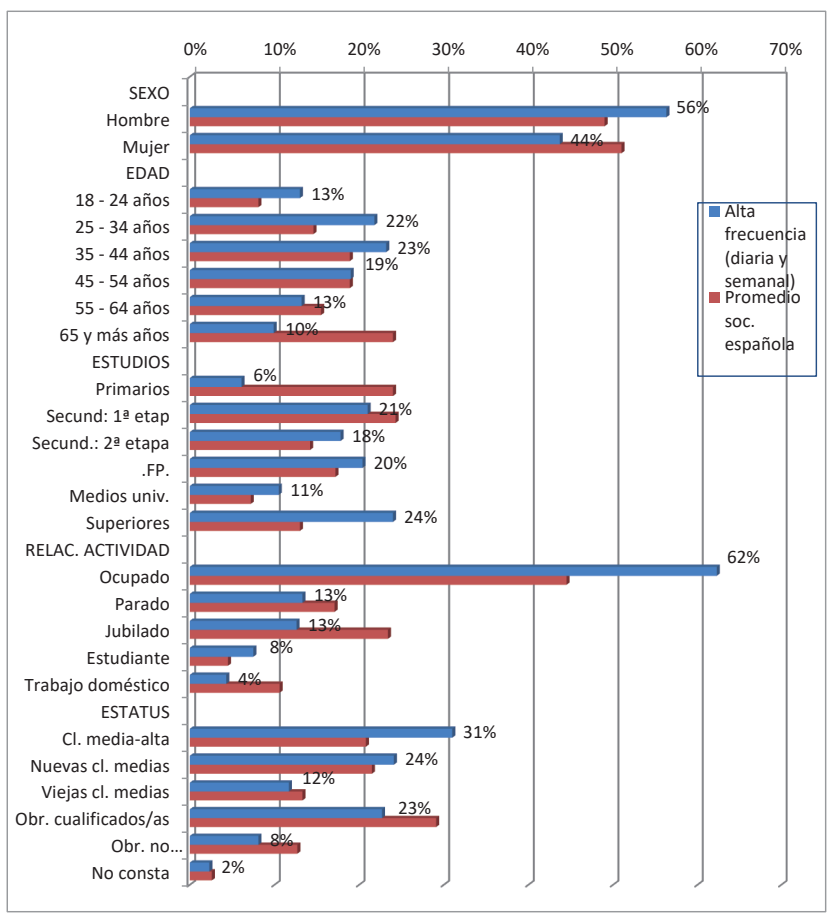

Todas las relaciones son significativas con un nivel de confianza superior al $99,9 \%$.

Fuente: elaboración propia con datos del Centro de Investigaciones Sociológicas, Barómetro de marzo 2017; estudio 3170.

Las variables asociadas a comportamientos de vida cotidiana (Gráfico 3) ayudan a concretar el perfil del comensal extradoméstico (con una alta y media frecuencia de consumo fuera del hogar). La práctica deportiva de varias veces por semana supera a la de la media de la población, son también más madrugadores que el conjunto de la sociedad española (el $31 \%$ se levantan antes de las 7 de la mañana), y expresan una mejor autopercepción de la propia salud: el 18\% la considera muy buena y el 66\% la define como buena, 
valores que descienden al 15 y $63 \%$ en el conjunto de la sociedad española.

Gráfico 3. Hábitos de las personas que comen en restaurantes/cafeterías con una frecuencia diaria (alta frecuencia) y semanal (frecuencia media), y distribución de la población española.

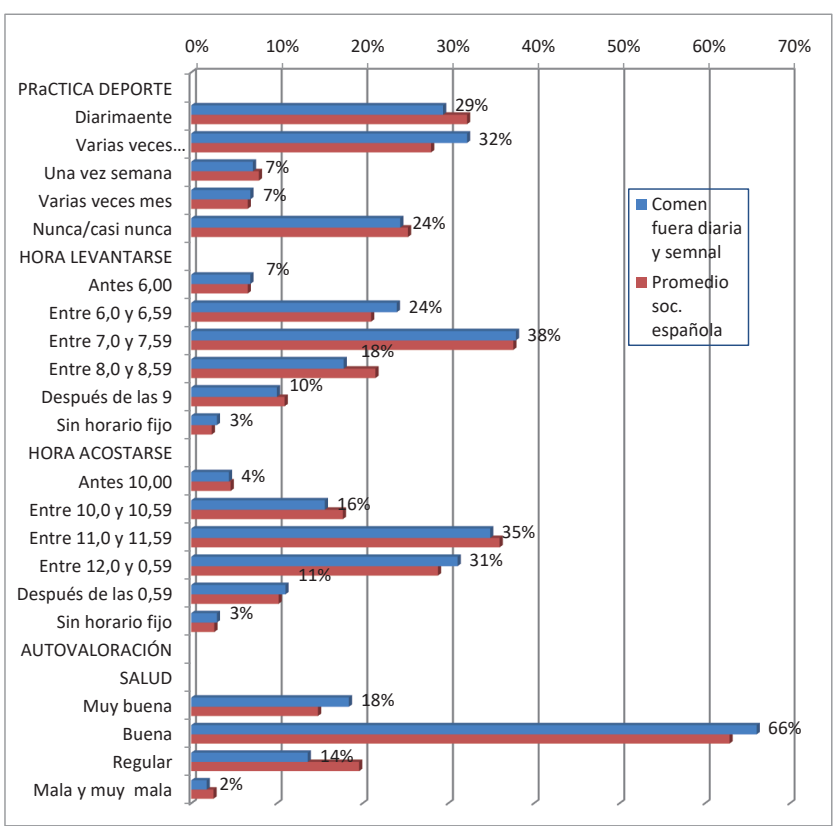

Todas las relaciones son significativas con un nivel de confianza superior al $99,9 \%$.

Fuente: Elaboración propia con datos del Centro de Investigaciones Sociológicas, Barómetro de marzo 2017; estudio 3170.

Además, se trata de un comensal que realiza un promedio de 3,6 ingestas diarias (no mostrado en el gráfico), considerado conjuntamente desayunos, comidas, cenas y cualquier comida tomada en otro momento. Este número es ligeramente inferior -pero significativo- al de la media de la población (3,7 comidas diarias).

Existen también diferencias en la frecuencia de consumo de algunos alimentos (gráfico 4). Ahí se constata que el $12 \%$ de la población española come carne a diario, y un $68 \%$ varias veces a la semana; porcentajes que aumentan notablemente en el colectivo de españoles que come fuera del hogar, confirmándose un consumo diario de carne del 14\% y semanal del $71 \%$.

La ingesta de pescado no muestra diferencias relevantes entre la población general españolas y aquellos consumidores con perfil extradoméstico. Únicamente destacar que estos últimos presentan un mayor consumo de pescado (61\% varias veces a la semana y $27 \%$ una vez a la semana), porcentajes ligeramente superiores al conjunto de la población.

El consumo de huevos es similar entre ambos colectivos, destacando únicamente que los que comen fuera presentan un consumo ligeramente superior, diferencia de $1,7 \%$ puntos superior al conjunto de la población. El consumo de leche y de cereales y no presentan diferencias entre los comensales extradomésticos y el resto de la población.

Gráfico 4: Frecuencia de consumo de determinados alimentos (en porcentajes).

Población general

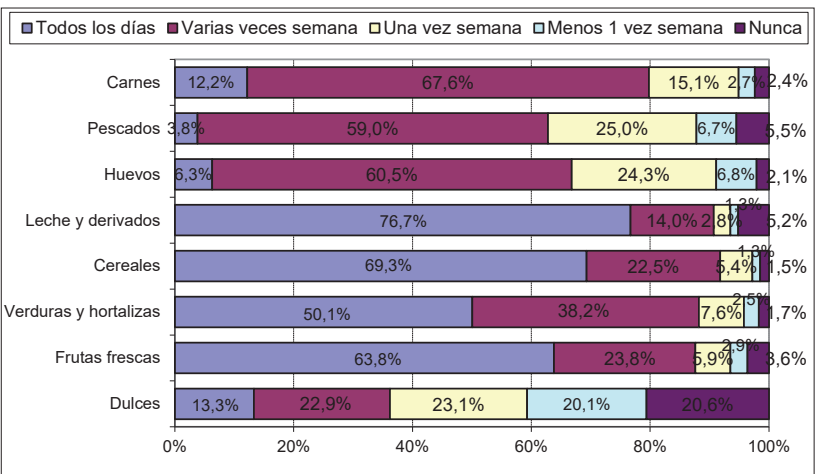

Consumidores que comen en restaurantes y cafeterías con alta y media frecuencia

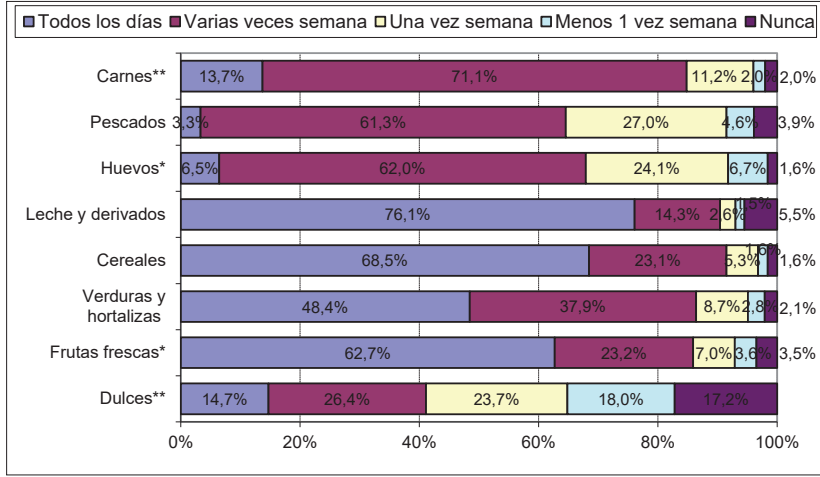

$* 0,05$

** $<0,05$

Fuente: elaboración propia con datos del Centro de Investigaciones Sociológicas, Barómetro de Marzo 2017, estudio 3170.

No ocurre lo mismo cuando se consideran los alimentos frescos, verduras-hortalizas y futas, y mucho menos con los dulces. En las verduras y hortalizas la mitad de la población española declara un consumo diario, y el $38 \%$ de varias veces por semana, porcentajes 
que aumentan en el consumo de frutas al 64\% (todos los días) y 24\% (varias veces a la semana). Véase en el gráfico 4 que los que comen fuera de casa reducen ligeramente el consumo de ambos productos.

Este cambio de hábitos en el postre tiene su alternativa en un aumento del consumo de dulces, consumidos a diario por un $15 \%$ de los que fuera a diario, y por un $26 \%$ de los que lo hacen varias veces a la semana. Es decir, el consumo de dulces es muy superior entre los comensales extradomésticos con alta y media frecuencia.

En definitiva, los que comen fuera en restaurantes/ cafeterías presentan un mayor consumo de carme, más huevos, más dulces y menos productos frescos (verduras-hortalizas y frutas). Estas pautas son más acusadas entre los que comen fuera de casa todos los días, frente a quienes lo hacen alguna vez a la semana y podrían llevar a la sospecha mayores problemas de sobrepeso y obesidad entre este colectivo, sin embargo, esta hipótesis ha sido rechazada en estudios españoles recientes (Díaz Méndez y García Espejo 2018; Fundación Mapfre, 2015).

En base a estos hallazgos pudiera plantearse como hipótesis de trabajo que este cambio en la dieta generará -"para compensar"- una mayor práctica deportiva. Sin embargo, la frecuencia de comer fuera está asociada a una menor práctica deportiva, en la medida que el 29\% de los que más comen fuera hacen deporte 'todos los días', dato que asciende al 32\% en el conjunto de la sociedad española (Gráfico 3).

\section{GRADO DE INFLUENCIA DE LAS VARIABLES QUE DETERMINAN EL HÁBITO DE COMER FRECUENTEMENTE FUERA DEL HOGAR (DIARIO Y SEMANALMENTE)}

En el apartado anterior se ha definido el perfil del colectivo que más come en restaurantes y cafeterías, tanto considerando los rasgos sociodemográficos (Gráfico 2) como los diferentes hábitos de vida cotidiana (Gráfico 3 y 4). Tras esta primera descripción es el momento de conocer los factores que más determinan el perfil de comensal extradoméstico utilizando para ello el análisis de segmentación de modo exploratorio (Escobar, 2007). El objetivo, en primer lugar, es conocer qué variables influyen en el hábito de comer frecuentemente en restaurantes y cafeterías; es decir, cuáles de las variables incluidas en los gráficos 2 y 3 presentan más influencia a la hora de explicar este hábito. Con este análisis no solo se identifican las variables influyentes, sino que, además, estas se presentan de forma jerarquizada, esto es, se muestra en primer lugar la variable más influye, la segunda más influyente, la tercera, y así hasta que no existen variables sin influencia en la frecuencia de comida extradoméstica.

Además, el empleo de la técnica permite conocer cómo se comportan los diferentes valores de cada una de las variables a la hora de "potenciar" o "reducir" una determinada situación. Pero no solamente se detecta la influencia de cada variable, sino que permite localizar el efecto de la interacción, esto es, cómo determinadas combinaciones de categorías (de variables) presentan efectos más extremos en la variable dependiente. El objetivo, en definitiva, es conocer qué variables influyen en la frecuencia de comer frecuentemente fuera del hogar, la agrupación de valores de los predictores, y la búsqueda de interacción (Escobar, 2007).

La necesidad de contar con una gran muestra, requisito imprescindible para el desarrollo de esta técnica, ha llevado a interrumpir el proceso de segmentación en el momento que el grupo alcanza los 200 casos, o cuando no se pueda generar un subgrupo de, al menos, 100 casos.

El análisis de segmentación con las 12 variables del cuadro 1 produce 13 nodos terminales (Gráfico 5 y Tabla 1). El nodo de partida (o nodo "0"), mostrado en la parte superior, informa que el $38,4 \%{ }^{3}$ de la muestra $(11,5 \%$ de alta frecuencia $+26,6 \%$ de media $)$ come en restaurantes y cafeterías con una frecuencia diaria o semanal, siendo el nivel de estudios la variable que produce más diferencias. El colectivo sin estudios, apenas un 5,2\% de la muestra, es el segundo que menos come fuera de casa $(7,8 \%)$. Le siguen las personas con estudios primarios (el 18\% de la muestra) con un bajo consumo fuera de casa.

Centrados en el colectivo con estudios primarios, la edad es la siguiente variable que segmenta el término dependiente, seguida del sexo. El consumo fuera de casa desciende notablemente en las personas mayores de 65 años: el 3,6\% de las mujeres mayores de 65 años

3 La diferencia en cinco décimas, respecto al porcentaje señalado en el primer párrafo del cuarto epígrafe tiene su explicación en la falta de respuesta de determinadas variables. 
Gráfico 5: Árbol de segmentación con los aspectos más determinantes en comer en restaurantes/cafeterías con una frecuencia diaria y semanal.

Parte izquierda del árbol

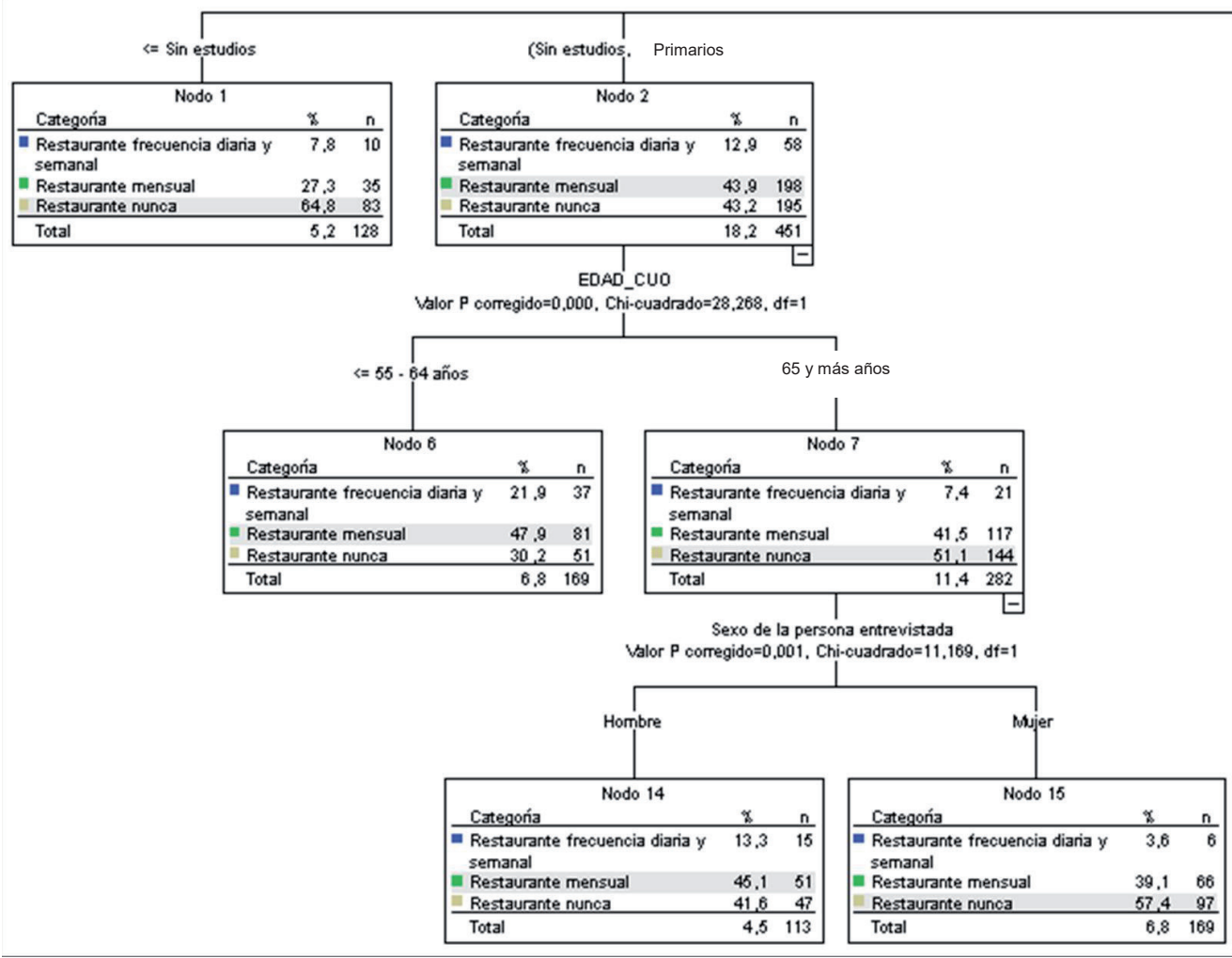

Parte central

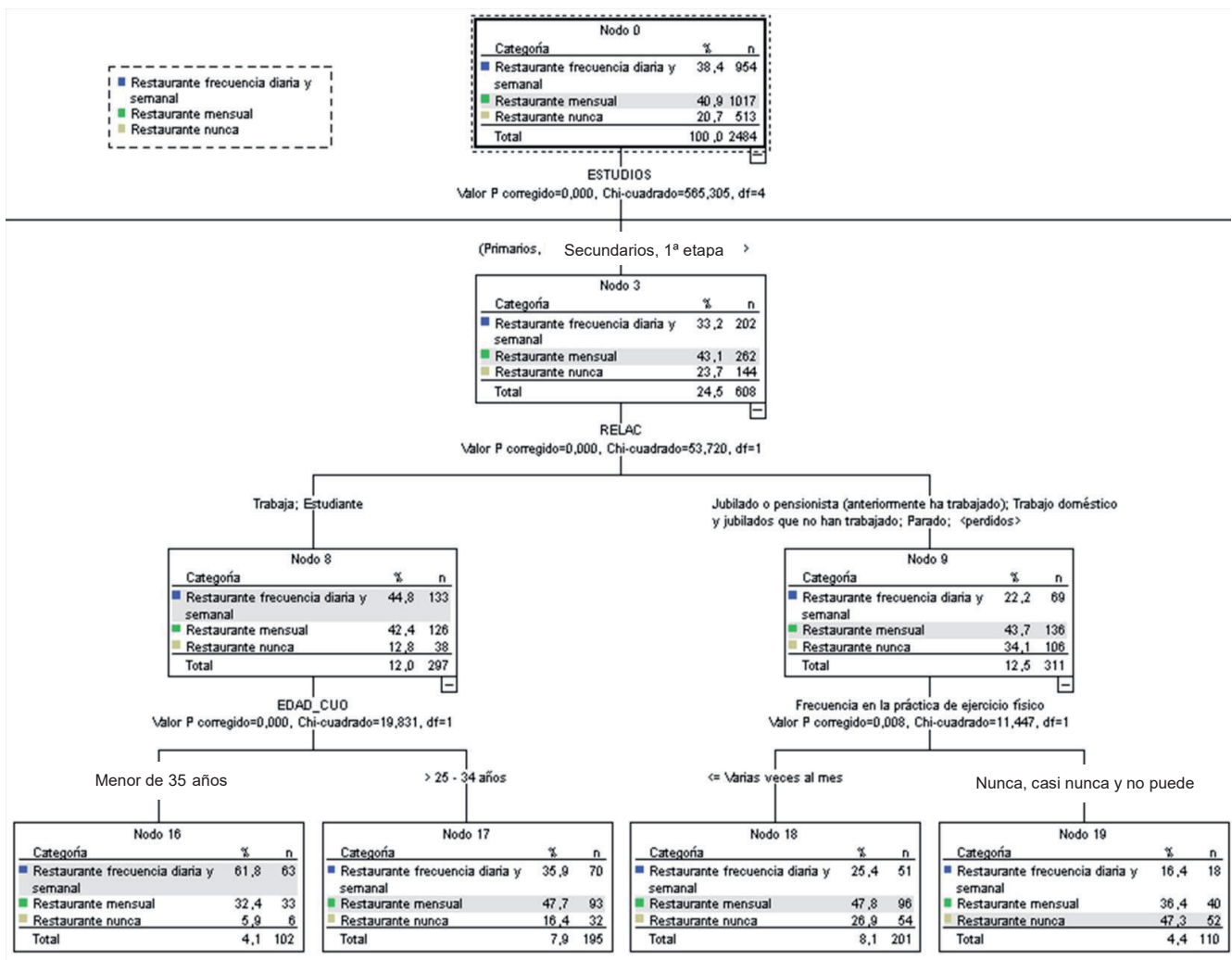


Parte derecha del árbol

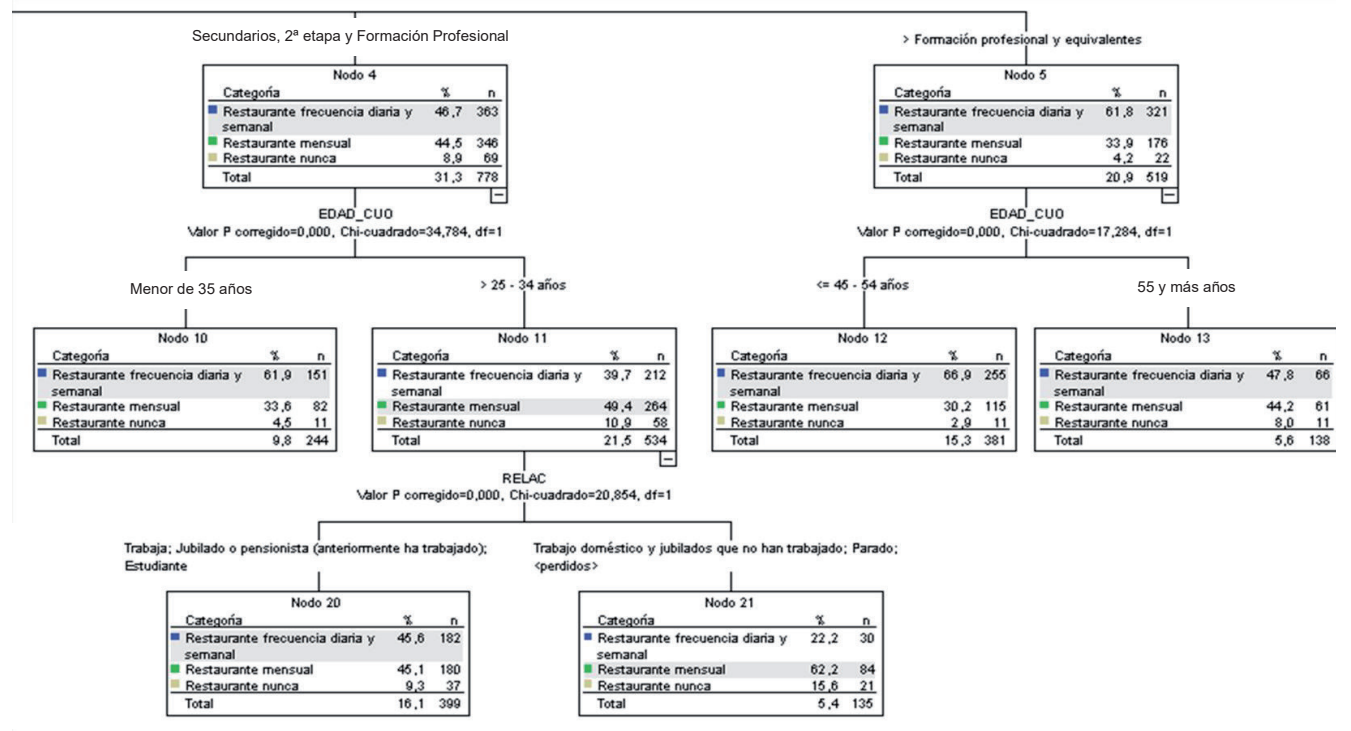

Fuente: Elaboración propia con datos del CIS, Barómetro de Marzo 2017; estudio 3170.

que tienen estudios primarios come en restaurantes y cafeterías, porcentaje que aumenta al 13,3\% en el caso de los hombres. Así, los colectivos con menor consumo extradoméstico son las personas con niveles educativos bajos y mayor edad.

En la parte central del árbol aparecen situados los entrevistados con estudios secundarios de primera etapa, un $25 \%$ de la muestra, que es segmentada según la relación con la actividad y, posteriormente, por la edad. La primera variable agrupa por un lado a los ocupados y los estudiantes, que vuelven a ser segmentados por la edad: mayores y menores de 35 años. Un $62 \%$ de los menores de 35 años comen frecuentemente en restaurantes y cafeterías, mientras que los mayores presentan un consumo similar al promedio. Los entrevistados con estudios secundarios (de primera etapa) que están jubilados, parados o son trabajadores domésticos son segmentados según la práctica deportiva, diferenciando los que hacen y no hacen deporte, sin importar el grado de actividad. Un $25 \%$ de los que hacen deporte comen frecuentemente en restaurantes/cafeterías, cifra que desciende al 16\% entre los que no lo practican.

La parte derecha del árbol, dedicada a las personas con mayores niveles de estudios, diferencian los que han terminado los estudios secundarios de segunda etapa y/o formación profesional de los estudios universitarios.
Estos últimos, un $21 \%$ de la muestra, presentan las mayores tasas de comidas fuera, llegando al $67 \%$ en los menores de 55 años. Casi la mitad de los mayores de 55 años comen frecuentemente en restaurantes.

Los que han terminado estudios secundarios de segunda etapa o formación profesional son segmentados por la edad, diferenciando -de nuevo- los menores y mayores de 35 años. El 62\% de los menores de 35 años come en restaurantes frecuentemente. Respecto a los mayores, son clasificados según su relación con la actividad, en este caso diferenciando los ocupados, jubilados y estudiantes del resto. El primer grupo presenta una tasa de consumo fuera ligeramente superior al promedio $(45,6 \%)$, que se reduce a la mitad en el caso de los parados y los trabajadores domésticos no remunerados. No deja de resultar sorprende la escasa influencia del estatus social, muy unida a los ingresos, variable "determinante" según algunas investigaciones (entre otras, Ramos Truchero y Castaño Suárez 2018; Fundación Mapfre, 2015).

Obsérvese que siete de las variables utilizadas no aparecen en el gráfico 5, situación producida por su escaso poder explicativo en la conducta objeto de estudio. El estatus del hogar -dentro de las variables sociodemográficas- y el horario habitual para levantarse y acostarse, el número habitual de comidas, la frecuencia 
de consumo de diferentes alimentos, y la valoración de su estado de salud en general -dentro de las variables de comportamiento- tienen un menos poder explicativo que el nivel de estudios, edad, relación con la actividad y la práctica deportiva.

Esta clasificación, que permanece estable cuando se llevan a cabo varias estrategias de validación, produce un $75,2 \%$ de clasificaciones correctas en la categoría objeto de estudio, y una reducción relativa del riesgo del 9,237 (Escobar, 2007).

En la tabla 1 se presentan los nodos terminales, ordenados según el porcentaje de personas de cada nodo que comen diaria y semanalmente en restaurantes y cafeterías. Más que esta cifra interesa el índice de ganancia, que es la presencia de la categoría objeto de estudio en cada uno de los nodos terminales. Así, la primera línea -nodo 12- se interpreta señalando que el $66,9 \%$ de los menores de 55 años con estudios universitarios comen con frecuencia diaria y semanal en restaurantes y cafeterías. Obsérvese la gran relación de los estudios elevados con comer fuera del hogar, altos estudios combinados con la edad en la mayor parte de los nodos situados en la parte superior, y con la relación con la actividad en dos de ellos (nodos 16 y 20). La última columna, calculada dividiendo la ganancia entre el porcentaje total de personas que comen en estos establecimientos $(66,9 / 38,4)$ indica "cuánto mayor" es el consumo de este colectivo respecto a la distribución muestral total.

Tabla 1. Tabla de ganancias.

\begin{tabular}{|c|c|c|c|c|c|c|c|}
\hline \multirow[b]{2}{*}{ Gananci } & \multirow[t]{2}{*}{$\begin{array}{l}\text { Variables definitorias de cada nodo } \\
\text { porcentaje (en el orden que aparecen) }\end{array}$} & \multicolumn{2}{|c|}{$\begin{array}{c}\text { NODO } \\
\mathrm{N}^{\mathrm{o}} \text { casos y }\left(\mathrm{N}^{\mathrm{o}} \mathrm{y}\right. \\
\text { porcentaje respecto } \\
\text { comen fuera })\end{array}$} & \multicolumn{2}{|c|}{$\begin{array}{l}\text { Ratio respecto al } \\
\text { tamaño del nodo }\end{array}$} & \multirow[t]{2}{*}{$\begin{array}{l}\text { GANANCIA } \\
\text { Ratio comen en } \\
\text { rest-cafet. entre de } \\
\text { tamaño nodo }\end{array}$} & \multirow[t]{2}{*}{$\begin{array}{l}\text { Índice } \\
\text { de } \\
\text { Ganancia }\end{array}$} \\
\hline & & & & & & & \\
\hline 12 & $\begin{array}{l}\text { Estudios universitarios, } \\
\text { menor de } 55 \text { años }\end{array}$ & 381 & $15,3 \%$ & 255 & $26,7 \%$ & $66,9 \%$ & $174,3 \%$ \\
\hline 10 & $\begin{array}{l}\text { Estudios secundarios } 2^{\mathrm{a}} \\
\text { etapa, menor de } 35 \text { años }\end{array}$ & 244 & $9,8 \%$ & 151 & $15,8 \%$ & $61,9 \%$ & $161,1 \%$ \\
\hline 16 & $\begin{array}{l}\text { Estudios secundarios } 1^{\text {a }} \text { etapa, ocupados } \\
\text { y estudiantes, menor de } 35 \text { años }\end{array}$ & 102 & $4,1 \%$ & 63 & $6,6 \%$ & $61,8 \%$ & $160,8 \%$ \\
\hline 13 & $\begin{array}{l}\text { Estudios universitarios, } \\
\text { mayor de } 55 \text { años }\end{array}$ & 138 & $5,6 \%$ & 66 & $6,9 \%$ & $47,8 \%$ & $124,5 \%$ \\
\hline \multirow[t]{2}{*}{20} & $\begin{array}{l}\text { Estudios secundarios } 2^{\text {a }} \text { etapa, } \\
\text { mayor } 35 \text { años, trabajadores, } \\
\text { jubilados y estudiantes }\end{array}$ & 399 & $16,1 \%$ & 182 & $19,1 \%$ & $45,6 \%$ & $118,8 \%$ \\
\hline & \multicolumn{5}{|c|}{ Sumatorio del \% de población en los 5 primeros nodos } & & $50,9 \%$ \\
\hline 17 & $\begin{array}{l}\text { Estudios secundarios } 1^{\text {a }} \text { etapa, ocupados } \\
\text { y estudiantes, } 35 \text { y más años }\end{array}$ & 195 & $7,9 \%$ & 70 & $7,3 \%$ & $35,9 \%$ & $93,5 \%$ \\
\hline 18 & $\begin{array}{l}\text { Estudios secundarios } 1^{\mathrm{a}} \text { etapa, } \\
\text { jubilados, parados y trabajo } \\
\text { doméstico, práctica deportiva }\end{array}$ & 201 & $8,1 \%$ & 51 & $5,3 \%$ & $25,4 \%$ & $66,1 \%$ \\
\hline 21 & $\begin{array}{l}\text { Estudios secundarios } 2^{a} \text { etapa, } \\
\text { mayor } 35 \text { años, trabajo doméstico }\end{array}$ & 135 & $5,4 \%$ & 30 & $3,1 \%$ & $22,2 \%$ & $57,9 \%$ \\
\hline 6 & Estudios primarios, menos de 65 años & 169 & $6,8 \%$ & 37 & $3,9 \%$ & $21,9 \%$ & $57,0 \%$ \\
\hline 19 & $\begin{array}{l}\text { Estudios secundarios } 1^{\mathrm{a}} \text { etapa, } \\
\text { jubilados, parados y trabajo } \\
\text { doméstico, nunca hacen deporte }\end{array}$ & 110 & $4,4 \%$ & 18 & $1,9 \%$ & $16,4 \%$ & $42,6 \%$ \\
\hline 14 & Estudios primarios, 65 y más años, varón & 113 & $4,5 \%$ & 15 & $1,6 \%$ & $13,3 \%$ & $34,6 \%$ \\
\hline 1 & Sin estudios & 128 & $5,2 \%$ & 10 & $1,0 \%$ & $7,8 \%$ & $20,3 \%$ \\
\hline 15 & Estudios primarios, 65 y más años, mujer & 169 & $6,8 \%$ & & $6,6 \%$ & $3,6 \%$ & $9,2 \%$ \\
\hline
\end{tabular}

Métodos de crecimiento: CHAID

Variable dependiente: (ordinal)

Fuente: elaboración propia con datos del Centro de Investigaciones Sociológicas, Barómetro de Marzo 2017 ; estudio 3170. 
En definitiva, las variables sociodemográficas -principalmente nivel de estudios y edad- presentan una mayor capacidad segmentadora que las relativas a los hábitos de vida cotidiana. Solo una de estas variables (frecuencia en la práctica de ejercicio físico) interviene en el modelo, y en un nivel muy inferior (lo que implica que afecta a un bajo número de entrevistados).

Respecto a la importancia de las variables predictoras, en los estudios secundarios de primera etapa interviene la relación con la actividad y la edad. Concretamente, en los estudios secundarios de segunda etapa y universitarios, el colectivo más numeroso ( $52 \%$ de los entrevistados), interviene la edad en primer lugar y la relación con la actividad y la edad, en segundo lugar.

A tenor de los resultados podría señalarse que es el nivel de estudios la variable más importante para diferenciar los perfiles de los colectivos de la sociedad española que come frecuentemente (diaria y semanal) en restaurantes y cafeterías. Le siguen en relevancia la edad y la relación con la actividad.

Resulta llamativo, por otro lado, el escaso (o nulo) poder segmentador de variables relacionadas con hábitos diarios y asentados como son las horas de sueño, las comidas realizadas diariamente, y la valoración de la salud.

\section{CONCLUSIONES}

En este trabajo se han analizado los factores que determinan el consumo alimentario fuera del hogar. También se ha descrito el perfil de comensal extradoméstico analizando las características sociodemográficas y los hábitos de vida cotidiana que explican este comportamiento, y que hacen de este colectivo un grupo particular.

Los resultados muestran un perfil de comensal extradoméstico que se distancia del español medio en varios aspectos: estamos ante un varón, de menos de 44 años y entre los que predominan las personas ocupadas de nivel educativo alto. Aunque se detecta un perfil diferenciado que le distancia del resto de la población, no estamos ante un comensal con unos hábitos drásticamente diferenciados del resto, pero si difieren en algunos hábitos alimentarios que pueden resultar significativos, pues consumen más carne, menos verduras y legumbres, menos frutas, más huevos y más dulces que el resto de la población. Todo ello hace que su dieta diaria presente hábitos menos saludables. Esto se acompaña de una práctica deportiva diaria menor que la de la mayoría de la población. Además, se levantan más tarde y se acuestan más tarde que la mayoría, por lo que las horas de sueño son menores. A pesar de estos rasgos, que podrían aproximarnos a unos hábitos menos saludables, los comensales extradomésticos tienen una autovaloración sobre su salud más positiva que la del resto de la población.

Diversos estudios han señalado que el gasto alimentario fuera del hogar tiene una relación directa con la renta, la ocupación y el nivel educativo. Ahora bien, lo que diferencia este trabajo de la investigación precedente es que aquí todas estas variables, junto con otras relativas a los hábitos saludables cotidianos, actúan simultáneamente para explicar el hábito de comer fuera de casa. Los resultados indican que el nivel educativo es la variable que tiene un mayor poder explicativo. La segunda variable con mayor capacidad explicativa es la edad, en las personas con estudios primarios, y en aquellos que tienen estudios secundarios de segunda etapa y más elevados. En el colectivo con estudios secundarios de primera etapa la relación con la actividad es la segunda variable más influyente.

En el tercer nivel de influencia aparecen diversas variables con menor poder explicativo: el sexo en las personas con estudios primarios con 65 y más años; la edad en los que tienen estudios secundarios de primera etapa y trabajan/estudian, la frecuencia de ejercicio físico en el resto de personas con estudios secundarios de primera etapa (jubilados, amas de casa y parados) y la relación con la actividad en entrevistados con más de estudios primarios de primera etapa y entre 25 y 34 años.

El estudio de los nodos terminales desvela que tener estudios secundarios y más elevados, y una edad inferior a los 55 años incrementa notablemente el comer diaria-semanal-mensualmente en restaurantes; incremento que es mayor en los colectivos de mayores estudios.

En definitiva, los resultados desvelan, en particular, el gran poder segmentador del nivel de estudios coincidiendo así con otros trabajos precedentes 
y reforzando la importancia de las variables sociodemográficas.

La educación tiene un peso relevante en la decisión de comer fuera, pero el peso de esta variable puede entenderse por la mejor posición ocupacional que permiten el nivel educativo, o bien por el carácter de distinción social que tiene la comida fuera de casa para los grupos sociales con mayor educación. ¿Podemos diferenciar si estamos ante un rasgo de distinción social relacionado con el ocio o ante un hábito instrumental de quienes ocupan las mejores posiciones laborales? En esencia iestamos ante una actividad estrictamente laboral o claramente recreativa marcada por el nivel de estudio?

Considerando que la comida doméstica es aquella "que es cocinada por un miembro del hogar e ingerida en el mismo hogar" (Díaz Méndez y Amparo Novo, 2017) y todo lo demás es comida extradoméstica, hay que pensar en la ambigüedad de su definición (Miller et al, 2014). ¿Todos habrán interpretado del mismo modo el término "comida fuera de casa"?, ¿consideran la comida en puestos ambulantes?, ¿y la comida en máquinas expendedoras?. A esto hay que añadir que la comida en cafeterías y restaurantes se ofrece en la misma respuesta cuando no se trata de la misma oferta de restauración. Preguntar conjuntamente por estos lugares, puede proporcionar resultados desajustados a la situación de consumo, no desvelando si estamos ante una comida laboral o de ocio, y por lo tanto, no permitiendo explicar plenamente el efecto del nivel educativo.

Las escasas pautas de diferenciación en cuanto a quienes comen fuera de casa, y la media nacional confirmada aquí, muestran que la alimentación española sigue un patrón similar dentro y fuera del hogar. Esto está indicando que en el entorno extradoméstico español no se come en restaurantes de comida industrializada, sino en espacios que ofertan una alimentación similar a la doméstica. El carácter lúdico u obligatorio podría detectarse preguntando por la diferencia entre la semana laboral y el fin de semana, algo que se ha constatado relevante en otras investigaciones (Díaz Méndez y García Espejo, 2017). Aquí no es posible saberlo.

Por otra parte, la desviación hacia el consumo de algunas ingestas fuera del hogar (como los dulces o la carne), puede estar indicando una práctica menos restrictiva que la doméstica, en la que se saltan las normas más fácilmente, y por ello más lúdica que obligatoria. La mejor valoración que hacen de la salud los comensales extradomésticos en nuestros resultados, puede ser un síntoma de la satisfacción que produce comer fuera. Sea por ocio o por trabajo, sea por distinción social o por obligación, todo apunta a que estamos ante una práctica alimentaria satisfactoria de la que se ven beneficiados en particular los colectivos de niveles educativos más alto.

Las relaciones sociales que conlleva la comida fuera del hogar remarcan su carácter voluntario y le imprime connotaciones positivas, incluso aunque comer fuera de casa sea una imposición marcada por el trabajo. La falta de datos sobre los motivos y las relaciones hace imposible distinguir si estamos ante un hábito obligatorio o voluntario y no desvela información acerca del carácter lúdico de la comida extradoméstica.

Finalmente, cabe matizar dos aspectos de los resultados: por un lado, el peso de la educación podría estar indicando que un mayor conocimiento, más información o mayor receptividad a los mensajes modifica las elecciones alimentarias y determina la alimentación fuera del hogar, sin embargo, también cabe suponer que esta variable está asociada a la renta, así, no sería tanto la formación como los ingresos que facilitan la cualificación, lo que explicaría las diferencias de comportamiento. Por otra parte, la confirmación de que las variables sociodemográficas son más explicativas que las variables relacionadas con los estilos de vida saludables puede estar indicando que aquellas marcan la alimentación extradoméstica, pero también que las variables de salud asociadas a la vida cotidiana no reflejan diferencias entre los comportamientos alimentarios extradomésticos y domésticos. Como se ha visto en otros estudios, los hábitos alimentarios en España, en uno y otro ámbito, no difieren ni en el tipo de comida ingerida, ni en los horarios, ni en la compañía (Díaz-Méndez y García Espejo, 2017). Estamos ante conductas muy enraizadas en la cultura alimentaria española cuyas normas de comportamiento muestran gran homogeneidad y cuyas diferencias se difuminan, dejando visibles solamente aquellas de carácter estructural que estratifican la sociedad tanto en este asunto, la alimentación, como en otros. 
Lamentablemente, y esto constituye una limitación del presente trabajo, no se puede dar respuesta a esta cuestión de manera concluyente, en especial por las limitaciones metodológicas de la encuesta empleada, pero se pueden aproximar algunas explicaciones.

Finalizar señalando las principales aportaciones de este trabajo, constatando el gran poder explicativo de las variables sociodemográficas, frente a las relacionadas con la vida cotidiana como las horas de sueño, el número de comidas realizadas en días laborables, el consumo de diferentes alimentos, la frecuencia de comidas fuera de casa, la práctica de ejercicio físico, y la autovaloración de su estado de salud. Aunque diversos estudios (entre otros, Abellan et al., 2016; Åkerstedt et al., 2019; Castro-Vazquez et al., 2007; Diener et al., 1999; Escobar et al., 2013; Estudio ALADINO, 2015; Ferrer-Cascales et al., 2019; Girón, 2010; Grao-Cruces et al, 2002; Marqueta se Salar et al., 2017; Serra Majem et al., 2000; Veenhoven, 2019) han destacado que se trata de variables que influyen en la salud de los individuos, el análisis conjunto de estas variables y de las variables sociodemográficas desvela una menor influencia de aquellas. En definitiva, el presente trabajo corrobora el perfil del comensal extradoméstico español, con una escasa influencia de las variables de la vida cotidiana.

\section{REFERENCIAS BIBLIOGRÁFICAS}

Abellán, G. B., Hidalgo, J. D. L. T., Sotos, J. R., López, J. L. T., y Jiménez, C. L. V. 2016. "Alimentación saludable y autopercepción de salud". Atención Primaria, 48(8), 535542. DOI. 10.1016/j.aprim.2015.12.001

Åkerstedt, Torbjörn; Ghilotti, F., Grotta, A., Zhao, H. A., Hans-Olov; Trolle-Lagerros, Y. y Bellocco, R. 2019. "Sleep duration and mortality. Does weekend sleep matter?, Journal of Sleep Research, 28 (1), 962-1105.

Asociación Nacional de Marcas de Restauración 2018. Datos económicos 1994-2016, http://marcasderestauracion.es/ sector/datos-economicos/, último acceso 14 de mayo de 2018.

Bojanic, D., y Xu, Y. 2006. "An investigation of acculturation and the dining-out behavior of Chinese living in the United States". International Journal of Hospitality Management 25: 211-226. https://doi.org/10.1016/j. ijhm.2005.06.002

Bourdieu, P. 1991. La distinción: criterio y bases sociales del gusto. Barcelona: Taurus

Callejo, J. 2017: "Eppur si muove... También la comida". Pp. 53-129, en Comer fuera de casa. Las opciones alimentarias de las nuevas relaciones sociales, Madrid: Icaria.

Castro-Vázquez, A., Espinosa-Gutiérrez, I., RodríguezContreras, P., y Santos-Iglesias, P. 2007. Relación entre el estado de salud percibido e indicadores de salud en la población española. International Journal of Clinical and Health Psychology, 7 (3), 883-898.

Centro de Investigaciones Sociológicas-CIS 2014. Barómetro de noviembre 2014, estudio 3045. Madrid: CIS. http://www.cis.es/cis/opencm/ES/2_bancodatos/ estudios/ver. jsp?estudio =14127\&cuestionario $=16939 \&$ muestra $=22493$, último acceso 28 de mayo de 2018.

Centro de Investigaciones Sociológicas-CIS 2017. Barómetro de marzo 2017, estudio 3170. Madrid: CIS.http://www.cis.es/ cis/opencm/ES/2_bancodatos/estudios/ver.jsp?estudio= $14333 \&$ cuestionario $=17196 \&$ \&muestra $=23847$, último acceso 28 de mayo de 2018.

Centro de Investigaciones Sociológicas-CIS (año desconocido): Nota de investigación: actualización de las clasificaciones nacionales de ocupación y de actividad económica en los datos del CIS

Cheng S.-L., Olsen W., Southerton D., Warde A. 2007. The changing practice of eating. Evidence from UK time diaries, 1975 and 2001. The British Journal of Sociology 58: 39-61.

Cullen, P. 1994. "Time, tastes and technology: the economic evolution of eating out". British Food Journal 96: 4-9. https://doi.org/10.1108/00070709410072445

Díaz-Méndez, C. y García-Espejo, I. 2013. La alimentación fuera del hogar en España. Clarificando la dualidad entre la obligación y el placer. XI Congreso Español de Sociología (grupo 28, Sociología de la Alimentación), Madrid 10-12 de julio.

Díaz-Méndez; C. y García-Espejo, I. 2018. "La relación entre la alimentación fuera del hogar y la obesidad: Un estudio sociológico del caso español". Revista Española de Sociología, 27: 251-266. DOI. 10.22325/fes/res.2018.41

Díaz-Méndez, C. y Novo Vázquez, M. A. 2017. "La alimentación fuera del hogar en España: un hábito alimentario arraigado y en expansión". Pp. 7-22, en Comer fuera de casa. Las opciones alimentarias de las nuevas relaciones sociales. Madrid: Icaria.

Díaz-Méndez, C. y García-Espejo, I. 2017. "Preguntas y respuestas para comprender la cultura alimentaria extradoméstica en España”. Pp. 41-52, en Comer fuera de casa. Las opciones alimentarias de las nuevas relaciones sociales, Madrid: Icaria.

Diener, E., Suh, E. M., Lucas, R. E., and Smith, H. L. 1999. "Subjective Well-Being: Three Decades of Progress". Psychological Bulletin, 125 (2), 276-302. DOI. 10.1037/0033-2909.125.2.276

Escobar, C., González Guerra, E., Velasco-Ramos, M., Salgado-Delgado, R., y Angeles-Castellanos, M. 2013. "La mala calidad de sueño es factor promotor de obesidad". Revista mexicana de trastornos alimentarios. 4(2), 133-142.

Escobar, M. 2007. El análisis de segmentación: técnicas y aplicaciones de los árboles de clasificación. Madrid: Centro de Investigaciones Sociológicas.

Escobar, M. 1998 "Las aplicaciones del análisis de segmentación: el procedimiento Chaid". Empiria 1: 1349. 
Estudio ALADINO. 2015. Estudio de Vigilancia del Crecimiento, Alimentación, Actividad Física, Desarrollo Infantil y Obesidad en España 2015. AECOSAN.

Ferrer-Cascales, R., Albaladejo-Blázquez, N., Ruiz-Robledillo, N., Clement-Carbonell, V., Sánchez-SanSegundo, M., and García-Pardo, N. 1998: "Variable de clase social del CIS". Investigación y Marketing, 61, 59-60.

Gofton, L. 1995. "Dollar rich and time poor? Some problems in interpreting changing food habits". British Food Journal 97: 11-16.

Girón, P. 2010. "Los determinantes de la salud percibida en España". Dissertation submitted at the Department of Nursing, Universidad Complutense de Madrid.

Grao-Cruces, A., Nuviala, A., Fernández-Martínez, A., PorcelGálvez, A. M., Moral-Gustafsson, K., and Sidenvall, B. 2002. Food-related health perceptions and food habits among older women. Journal of Advanced Nursing, 39(2), 164-173.

Instituto Nacional de Estadística-INE 2017. Encuesta de Presupuestos Familiares Base 2006. Madrid: Instituto Nacional de Estadística-INE.

Kass, Gary V. 1980. "An Exploratory Technique for Investigating Large Quantities of Categorical Data". Applied Statistical 29: 119-127. DOI: 10.2307/2986296

Magdison, J. y Vermut, J. K. 2005: "An extension of the CHAID tree-based segmentation algoritm to multiple dependent variables". Pp. 176-183, en Classification: the ubiquitous challenge. Heidelberg: Springer.

Marqueta de Salas, M., Rodríguez Gómez, L., Enjuto Martínez, D., Juárez Soto, J. J., y Martín-Ramiro, J. J. 2017. "Relación entre la jornada laboral y las horas de sueño con el sobrepeso y la obesidad en la población adulta española según los datos de la Encuesta Nacional de Salud 2012". Revista Española de Salud Pública, 91, 201703023.

McCracken, V. A., Brandt,J. A. 1987. "Household consumption of food-away-from-home: total expenditure and by type of food facility". American Journal of Agricultural Economics 69: 274-284. DOI: $10.2307 / 1242277$

Miller, K.; Willson, S., Chepp, V. y Padilla, J.L. 2014: Cognitive interviewing methodology, Hoboken: Wiley.

Mapfre, Fundación 2015. Alimentación y sociedad en la España del siglo XXI. Madrid: Fundación Mapfre y Universidad San Pablo CEU.

Martín Cerdeño, V. J. 2003. "El sector de la restauración en España. Situación y factores explicativos". Distribución y Consumo, 69: 5-26.

Martin Cerdeño, V.J. 2012. "Restauración y coyuntura económica". Distribución y Consumo 121: 61-71.

Martin Cerdeño, V.J. 2017: "La estructura del sector de la restauración en España: la oferta alimentaria”. Pp. 5374, en Comer fuera de casa. Las opciones alimentarias de las nuevas relaciones sociales, Madrid: Icaria.
Ministerio de Agricultura, Alimentación y Medio Ambiente 2016): Informe del consumo de alimentación en España 2016. Madrid: Ministerio de Agricultura, Alimentación y Medio Ambiente. http://www.mapama.gob.es/es/alimentacion/ temas/informe_del_consumo_de_alimentos_en_ Espana_2016_webvf_tcm30-386079.pdf, último acceso 15 de mayo.

Olsen, W. K., Warde, A. y Martens, 1. 2000). "Social differentiation and the market for eating out in the UK". International Journal of Hospitality Management 19: 173190. DOI: $10.1046 / j .1471-5740.2001 .00007 . x$

Rama, R. 1997. "Evolución y características de la alimentación fuera del hogar y del consumo de alimentos procesados en España”. Agricultura y sociedad 84: 107-140.

Ramos Truchero; G. y Castaño Suárez, E. 2018). "Comer fuera de casa en tiempos de crisis: austeridad y formas de resistencia". Revista Española de Sociología, 27: 219-236. DOI: https://doi.org/10.22325/fes/res.2018.39

Resa López, A. 2019. "A golpe de máquina. El vending se prepara para convivir con un nuevo competidor. La tienda automatizada". Distribución y Consumo, 156: 26-30.

Román González, M.V. y Levy Mangin, J.P. 2003. "Clasificación y segmentación jerárquica". Pp. 567-590, en Análisis Multivariable para las Ciencias Sociales, Madrid, Pearson.

Riley, M. 1994. "Marketing eating out: the influence of social culture and innovation". British Food Journal 96: 15-18. https://doi.org/10.1108/00070709410072463

Serra Majem L, Aranceta Bartrina J, Ribas Bar-ba L, Pérez Rodrigo C, García Closas R. 2000. "EstudioenKid: objetivos y metodología". En: Serra MajemL, Aranceta J, editores. Desayuno y equilibrio alimentario. Estudio enKid. Barcelona: Masson S.A.1-8.13.

Sonquist, J.A. y Morgan, J. N. 1964. Applied The detection of interaction effects. Ann Arbor: Survey Research Center monograph $n^{\circ}$ 35, Institute for Social Research, Universidad de Michigan.

Truninger M y Ramos V (2021). "Cambios en la comensalidad en Portugal durante la crisis" en La alimentación en el sur de Europa, de Díaz-Méndez y Cardon. (en prensa).

Veenhoven, R. 2019. "Will healthy eating make you happier? A research synthesis using an online findings archive". Applied Research in Quality of Life, 1-20.

Warde, A. y Martens L. 1998. "Eating out and the commercialization of mental life". British Food Journal 100 : 147-154. 3.

Warde A. y Martens L. 2000. Eating Out: social differentiation, consumption and pleasure. Cambridge: Cambridge University Press.

Zaragoza-Martí, A. 2019. "Higher Adherence to the Mediterranean Diet is Related to More Subjective Happiness in Adolescents: The Role of Health-Related Quality of Life". Nutrients, 11(3), 698. DOI. 10.3390/ nu11030698 


\section{NOTAS BIOGRÁFICAS}

\section{VIDAL DÍAZ DE RADA}

Es catedrático de Sociología en el Departamento de Sociología y Trabajo Social en la Universidad Pública de Navarra. Entresusúltimas publicaciones destacan: "Internet como modo de administración de encuestas" (CIS, 2019) y "Multitarea en una encuesta online" (REIS, 2021).

\section{CECILIA DÍAZ-MÉNDEZ}

Es catedrática de Sociología en el Departamento de Sociología de la Universidad de Oviedo. Dirige el Grupo de Investigación en Sociología de la Alimentación, SOCIALIMEN, (http://grupos. uniovi.es/web/socialimen) en el que se desarrollan sus investigaciones y publicaciones.Resarchgate. https://www.researchgate.net/profile/Cecilia_DiazMendez. 